Victor Gonçalves Corrêa Neto ${ }^{1}$ Sandro Sperandei ${ }^{1}$

Luis Aureliano Imbiriba Silva ${ }^{1}$

Geraldo de Albuquerque Maranhão Neto ${ }^{2}$

Alexandre Palma

\section{Hipertensão arterial em adolescentes do Rio de Janeiro: prevalência e associação com atividade física e obesidade}

Arterial hypertension among adolescents in Rio de Janeiro: prevalence and association with physical activity and obesity
${ }^{1}$ Escola de Educação Física e Desportos, Centro d Ciências da Saúde,

Universidade Federal do Rio de Janeiro. Av. Brigadeiro Trompowski, Cidade Universitária.

21.941-590 Rio de Janeiro RJ Brasil.

victorgcn@hotmail.com

${ }^{2}$ Programa de Pós-

Graduação em Ciências da

Atividade Física,

Universidade Salgado de

Oliveira.
Abstract The scope of this study was to identify the prevalence of systemic arterial hypertension among adolescent students (aged 17-19 years) in the third and final year of high school in state schools in the municipality of Rio de Janeiro, and to investigate associations between systemic arterial hypertension and obesity and physical activity levels. Data on arterial pressure, body mass index and physical activity were gathered. The analysis included 854 individuals. Descriptive statistical analysis was applied to the sample, along with a Poisson regression model to determine the impact of the variables on the prevalence of systemic arterial hypertension. The prevalence of systemic arterial hypertension was 19.4\%. Male sex, overweight and obesity presented significant positive associations with systemic arterial hypertension $(p<0.05)$, while physical activity was not shown to be significantly associated with systemic arterial hypertension $(p>0.05)$. The nature of these relationships should be interpreted in the light of reflection and not of passive labeling based on hegemonic concepts.

Key words Arterial hypertension, Obesity, Physical activity, Adolescents
Resumo O objetivo do estudo foi identificar a prevalência de hipertensão arterial sistêmica em adolescentes estudantes do $3^{\circ}$ ano do ensino médio de escolas públicas estaduais dentro do município do rio de Janeiro com idade entre 17 e 19 anos, bem como investigar as associações de hipertensão arterial sistêmica com obesidade e níveis de atividade física. Dados de pressão arterial, indice de massa corporal e atividade física, foram coletados. A amostra foi composta por 854 indivíduos, a estatística descritiva foi aplicada na amostra, bem como um modelo de regressão de Poisson para determinar o impacto das variáveis na prevalência de hipertensão arterial sistêmica. A prevalência de hipertensão arterial sistêmica foi de 19,4\%. Sexo masculino, sobrepeso e obesidade se associaram positiva e significativamente com hipertensão arterial sistêmica $(p<0,05)$, já atividade física não mostrou associação significativa com hipertensão arterial sistêmica $(p>0,05)$. A natureza dessas relações deveria ser interpretada a luz da reflexão e não de rotulações passivas embasadas em conceitos hegemônicos.

Palavras-chave Hipertensão arterial, Obesidade, Atividade física, Adolescentes 


\section{Introdução}

A hipertensão arterial sistêmica (HAS) se destaca com notável relevância dentre os problemas de saúde pública em âmbito mundial ${ }^{1}$. Tal fato se alicerça na premissa de que a prevalência dessa enfermidade vem aumentando ${ }^{2}$, bem como na perspectiva de que níveis pressóricos elevados têm forte impacto no aumento de risco para doenças cardiovasculares ${ }^{3}$.

O aumento na prevalência de HAS parece não se restringir a população adulta. A literatura advoga que tal quadro atinge também adolescentes $^{4}$. Diante do presente contexto transparece a necessidade do diagnóstico da HAS em fases precoces da vida do sujeito ${ }^{5}$.

A atividade física e a obesidade se mostram como tendo importante associação com a PA de jovens ${ }^{6}$. Tal afirmativa é ratificada tanto por estudos de cunho epidemiológico que tentam estabelecer tais relações através de associações estatísticas ${ }^{7}$, quanto por estudos de cunho fisiológico, como a revisão de Torrance et al. ${ }^{8}$, que aborda os mecanismos biológicos possivelmente atribuíveis a tais relações.

São escassos estudos sobre prevalência de HAS na população adolescente do Rio de Janeiro, bem como sua associação com obesidade e atividade física dentro do mesmo contexto etário. Ainda, parece existir pouca preocupação com o impacto sazonal que as variáveis podem sofrer quando da demora demasiada da coleta de dados.

Sendo assim, o presente estudo tem como objetivo identificar a prevalência de HAS em adolescentes, em uma amostra constituída por estudantes do $3^{\circ}$ ano do ensino médio de escolas públicas estaduais localizadas dentro do município do Rio de Janeiro, com idade entre 17 e 19 anos, bem como investigar as associações de HAS com obesidade e níveis de atividade física.

\section{Métodos}

\section{Tipo de estudo}

O estudo se caracteriza como do tipo transversal.

\section{Amostra}

A população foi composta por todos os estudantes do terceiro ano do ensino médio da rede estadual de ensino público matriculados em escolas dentro do município do Rio de Janeiro com idade entre 17 e 19 anos.
O cálculo do tamanho da amostra teve como referência o número total de alunos matriculados (73.500) no ensino médio, estudando em escolas públicas estaduais situadas dentro do município do Rio de Janeiro, dado este fornecido pela Secretaria Estadual de Educação (SEE). Para um erro amostral estipulado em três pontos percentuais, com nível de confiança de $95 \%$ e prevalência presumida de $25 \%$ de HAS a amostra foi estimada em 792 adolescentes. Precavendo-se de possíveis perdas, procurou-se levantar um número $10 \%$ maior e atingir um total de 871 adolescentes.

A distribuição do número de alunos por Coordenadoria Metropolitana foi estimada a partir dos valores percentuais que cada uma delas comportava, considerando o total de alunos matriculados na última série do ensino médio em escolas públicas estaduais localizadas dentro do município do Rio de Janeiro.

As escolas foram escolhidas de forma aleatória, através de sorteio, as turmas foram selecionadas de acordo com o maior contingente da faixa etária do estudo.

\section{Coleta de dados}

Inicialmente, o projeto foi submetido ao conselho de ética da instituição, após isso, o projeto foi encaminhado para a SEE, e esperada a devida autorização da mesma. Após autorização da SEE, realizou-se o contato com cada coordenadoria metropolitana dentro do município do Rio de Janeiro (Metropolitanas III, IV e X) e, posteriormente, as escolas foram visitadas mediante apresentação de autorização fornecida pela devida coordenaria metropolitana.

Foram coletados os seguintes dados: peso, estatura, PA, e respostas a um questionário sobre atividades físicas.

\section{Instrumentos}

A massa corporal foi mensurada através de uma balança digital da marca Camry ${ }^{\circledR}$, modelo EB 867, e a estatura foi medida através de um estadiômetro da marca $\mathrm{KaWe}^{\circledR}$. Para aferição da PA foi utilizado o monitor digital da marca Omron, modelo HEM 742, que atingiu grau A para mensuração tanto da pressão arterial sistólica (PAS), quanto da pressão arterial diastólica (PAD) em adolescentes, segundo os critérios da British Hypertension Society ${ }^{9}$. Para avaliar o nível de atividade física dos indivíduos foi aplicado um questionário do tipo self report, o questionário internacional de nível de ativi- 
dade física - IPAQ versão curta - que tem sido aceito para monitorar níveis habituais de atividade física em adolescentes de ambos os sexos com mais de 14 anos $^{10}$.

Dados a respeito da pratica de atividade físico-esportiva, do nível socioeconômico, de já ter medido a PA anteriormente e da participação em aulas de educação física escolar, também foram obtidos através de questionário do tipo self report.

\section{Procedimentos}

Em uma primeira visita à escola, o questionário foi respondido pelos estudantes. Em um segundo momento, os estudantes foram divididos em pequenos grupos e encaminhados a um local reservado para realização das mensurações. Após a primeira medida da PA ter sido realizada em um indivíduo, este retornava ao final da fila do grupo, permanecendo em repouso, até que todos tivessem sua primeira medida aferida. Posteriormente, o procedimento recomeçava com o grupo todo até que todos tivessem passado por duas mensurações. Após a coleta da PA, os indivíduos tiveram a massa corporal e a altura coletadas em medidas únicas.

O IMC foi obtido através da divisão da massa corporal em quilogramas pela estatura em metros ao quadrado. Os resultados foram classificados segundo os padrões sugeridos por Cole et al. ${ }^{11,12}$.

A PA foi aferida pelo método oscilométrico e o manguito foi utilizado considerando-se os critérios da American Heart Association ${ }^{13}$ e da Sociedade Brasileira de Cardiologia ${ }^{14}$ para circunferência do braço. Foram feitas duas aferições com espaço mínimo de dez minutos entre uma e outra, sendo que para efeito de análise foi considerado o menor valor, e para categorização, dentre o menor valor, a PA que ilustrava a cifra mais elevada, fosse ela a sistólica ou a diastólica.

A classificação para indivíduos a partir de 18 anos seguiu o seguinte padrão: "ótimo", indivíduos com a PA abaixo de $120 \mathrm{mmHg}$ e $80 \mathrm{mmHg}$ para sistólica e diastólica, respectivamente; "normal" indivíduos com a PA entre 120 mmHg e 129 $\mathrm{mmHg}$ e $80 \mathrm{mmHg}$ e $84 \mathrm{mmHg}$, respectivamente, para sistólica e diastólica; "normal alta" sujeitos com a PA sistólica e diastólica, respectivamente, entre $130 \mathrm{mmHg}$ e $139 \mathrm{mmHg}$ e $85 \mathrm{mmHg}$ e $89 \mathrm{mmHg}$; e por fim, "hipertensos" sujeitos com a PA igual ou acima de $140 \mathrm{mmHg}$ e $90 \mathrm{mmHg}$ para sistólica e diastólica, respectivamente ${ }^{15}$. A classificação para indivíduos com 17 anos seguiu o padrão sugerido para crianças e adolescentes pela American Academy of Pediatrics ${ }^{5}$, considerando "normotensos" indivíduos com a PA abaixo do percentil 90, "limítrofes" indivíduos com a PA entre percentis 90 e 95 ou se a PA excedesse $120 / 80 \mathrm{mmHg}$, ficando menor que percentil 90 até menor que percentil 95 . Foram considerados "hipertensos" indivíduos situados acima do percentil 95. Para efeito da análise, a amostra foi dividida em dois grupos a saber: hipertensos, que incluiu os indivíduos de acordo com os pontos de corte para HAS como descrito acima, e não hipertensos, que compreendeu os indivíduos localizados em qualquer estrato das cifras pressóricas que não caracterizassem HAS.

Quanto ao nível de atividade física, os sujeitos foram classificados em muito ativo, ativo, irregularmente ativo "a" ou "b" e sedentário como sugere o nível de classificação de atividade física segundo o IPAQ.

\section{Tratamento estatístico}

Após a análise descritiva da amostra utilizada, foi desenvolvido um modelo de regressão de Poisson para determinar o impacto das variáveis na prevalência de hipertensão. Iniciou-se com um modelo completo, utilizando como variáveis preditivas o índice de desenvolvimento humano (IDH) do bairro onde a escola estava localizada, a coordenadoria a que a escola pertencia, o sexo, a classificação pelo IMC, a classe econômica do indivíduo, seu status relatado de atividade física e a sua classificação pelo IPAQ. A variável dependente foi a classificação da pressão arterial (não hipertenso $x$ hipertenso). As variáveis não significativas $(p>0,05)$ foram retiradas uma a uma, até que o modelo mais parcimonioso fosse obtido. A razão de verossimilhança e a análise dos resíduos foram utilizadas na avaliação do modelo final. As análises foram executadas no software R, versão 2.14.

Todos os sujeitos de pesquisa foram devidamente informados sobre o propósito e os métodos da investigação científica, bem como, sobre a instituição responsável e o caráter voluntário e sigiloso da participação de cada um. Antes do preenchimento do questionário, os responsáveis ou sujeitos de pesquisa maiores de idade leram o Termo de Consentimento Livre e Esclarecido, enquanto os sujeitos de pesquisa menores de idade leram o Termo de Assentimento Livre e Esclarecido. Após a dupla concordância, os sujeitos de pesquisa participaram, voluntariamente, da pesquisa, conforme tem sido sugerido pela Re- 
solução 196/96 ${ }^{16}$ do Conselho Nacional de Saúde para experimentos com seres humanos. O estudo foi aprovado pelo Comitê de Ética em Pesquisa com Seres Humanos do Hospital Universitário Clementino Fraga Filho da Universidade Federal do Rio de Janeiro, em conformidade com a declaração de Helsinki.

\section{Resultados}

O processo de coleta de dados teve a duração de três meses. Ao final do processo de coleta de dados, se atingiu um $n$ amostral de 884 indivíduos com dados para análise. Desses, durante a tabulação, 30 foram excluídos por falta de relato de informações pessoais de cunho imprescindível para caracterização da amostra. Um $n$ de 854 adolescentes, 510 meninas e 344 meninos, foi o quantitativo final da amostra a ser analisada. Tais perdas não invalidam o caráter probabilístico da amostra, já que o cálculo amostral sugeriu um levantamento de dados de 792 indivíduos, e nosso $n$ final ficou mais de $7 \%$ acima de tal valor. As características do grupo avaliado $(\mathrm{n}=854)$ estão ilustradas na Tabela 1.

No que diz respeito a prevalência de HAS, a Tabela 2 ilustra os valores percentuais de adolescentes nas categorias ótima, normal, normal alta

Tabela 1. Valores médios referentes às características do grupo amostral.

\begin{tabular}{lr}
\hline \multicolumn{1}{c}{ Característica } & \multicolumn{1}{c}{$\begin{array}{c}\text { Média } \pm \\
\text { Desvio padrão }\end{array}$} \\
\hline Idade (Anos) & $17,7 \pm 0,68$ \\
Altura (Metro) & $1,66 \pm 0,09$ \\
Peso (Quilograma) & $62,2 \pm 13,02$ \\
IMC (Índice de Massa Corporal) & $22,5 \pm 4,02$ \\
Pressão Arterial Sistólica (mmHg) & $126 \pm 15,13$ \\
Pressão Arterial Diastólica (mmHg) & $74 \pm 9,3$
\end{tabular}

Tabela 2. Prevalência de hipertensão arterial entre os estudantes do ensino médio investigados.

\begin{tabular}{lcc}
\hline & \multicolumn{2}{c}{ Total } \\
\cline { 2 - 3 } Classificação da pressão arterial & n & $\%$ \\
\hline Ótima & 214 & 25,1 \\
Normal & 303 & 35,5 \\
Normal alta & 171 & 20,0 \\
Hipertensão & 166 & 19,4 \\
\hline
\end{tabular}

e hipertensão. Deve-se sublinhar que a categoria ótima só inclui indivíduos com 18 e 19 anos, já que a categorização sugerida para os sujeitos de 17 anos, não inclui tal faixa de classificação. Sendo assim, de uma forma geral pode-se dizer que no grupo investigado $19,4 \%$ dos indivíduos estão inclusos no grupo hipertensos, enquanto $80,6 \%$ dos indivíduos estão inclusos no grupo não hipertensos.

A razão de prevalência quando empregada em um modelo univariado, não foi capaz de identificar qualquer associação entre "prática de atividade física desportiva", "tempo de prática de atividade física desportiva" "nível de atividade física", "participação em aulas de educação física escolar", "índice de desenvolvimento humano (IDH)", "região da escola", "classe socioeconômica" "já ter medido a PA antes" e "peso abaixo do normal" com HAS ( $\mathrm{p}>0,05)$. Antagonicamente, "sobrepeso", "obesidade" e "sexo masculino" se associaram positiva e significativamente $(\mathrm{p}<$ 0,05) com HAS (Tabela 3 ).

As variáveis tidas com associação significantes no modelo univariado foram então analisadas sob o modelo multivariado. Todas as associações se mantiveram significativas $(p<0,05)$ (Tabela 4).

\section{Discussão}

O estudo constatou uma prevalência de HAS de $19,4 \%$, valor muito acima da expectativa para referida faixa etária, que seria em torno de $5 \%{ }^{17}$.

A mensuração da PA em uma única ocasião poderia justificar tal ocorrência, já que essa estratégia não é a recomendada para diagnosticar HAS $^{5}$. No entanto vale contra-argumentar que outros estudos que também realizaram medidas em uma única oportunidade, encontraram baixa prevalência de HAS em jovens ${ }^{18-20}$. Isso faz transparecer, que se medidas em múltiplas ocasiões podem ter um efeito redutor na prevalência de $\mathrm{HAS}^{21}$, medidas em um único momento podem, se não retratar valores literais em jovens ou adolescentes, ao menos apontar uma tendência ou um ponto de partida com relação às características pressóricas de determinada população.

A presente investigação não é a única realizada na região Sudeste que denotou grande número de indivíduos com padrões de PA elevados. Ribeiro et al. ${ }^{22}$, por exemplo, conduziram sua investigação em jovens de Belo Horizonte, Minas Gerais, e registraram 12\% de sua população com padrões de PA alterados. 
Tabela 3. Fatores de risco associados à prevalência de hipertensão arterial entre os estudantes do ensino médio investigados (modelo univariado).

\begin{tabular}{|c|c|c|c|c|c|c|c|}
\hline \multirow[t]{2}{*}{ Variáveis } & \multicolumn{2}{|c|}{ Não hipertensos } & \multicolumn{2}{|c|}{ Hipertensos } & \multirow[b]{2}{*}{$\mathbf{R P}$} & \multirow[b]{2}{*}{ IC } & \multirow[b]{2}{*}{ p } \\
\hline & $\mathbf{n}$ & $\%$ & $\mathbf{n}$ & $\%$ & & & \\
\hline \multicolumn{8}{|l|}{ Sexo $^{*}$} \\
\hline Meninas & 412 & 59,9 & 68 & 41 & ref. & ref. & ref. \\
\hline Meninos & 276 & 40,1 & 98 & 59 & 1,85 & $1,36-2,52$ & 0,000 \\
\hline \multicolumn{8}{|c|}{ Já havia medido a pressão arterial antes } \\
\hline $\operatorname{Sim}$ & 404 & 58,7 & 102 & 61,4 & ref. & ref. & ref. \\
\hline Não & 284 & 41,3 & 64 & 38,6 & 0,91 & $0,67-1,25$ & 0,565 \\
\hline \multicolumn{8}{|c|}{ Pratica atividade física desportiva } \\
\hline Sim & 389 & 56,5 & 105 & 63,3 & ref. & ref. & ref. \\
\hline Não & 299 & 43,5 & 61 & 36,7 & 0,80 & $0,58-1,09$ & 0,159 \\
\hline \multicolumn{8}{|c|}{$\begin{array}{l}\text { Tempo semanal de prática de atividade } \\
\text { física desportiva }\end{array}$} \\
\hline Acima de 300 minutos & 185 & 26,9 & 59 & 35,5 & ref. & ref. & ref. \\
\hline De 150,1 a 300 minutos & 110 & 16 & 24 & 14,5 & 0,74 & $0,46-1,19$ & 0,215 \\
\hline De 90,1 a 150 minutos & 54 & 7,8 & 12 & 7,2 & 0,75 & $0,40-1,40$ & 0,368 \\
\hline Até 90 minutos & 40 & 5,8 & 10 & 6,0 & 0,83 & $0,42-1,62$ & 0,579 \\
\hline Não faz & 299 & 43,5 & 61 & 36,7 & 0,70 & $0,49-1,00$ & 0,051 \\
\hline \multicolumn{8}{|c|}{$\begin{array}{l}\text { Nível de prática de atividades físicas } \\
\text { segundo o IPAQ }\end{array}$} \\
\hline Muito Ativos & 167 & 24,3 & 42 & 25,3 & ref. & ref. & ref. \\
\hline Ativos & 372 & 54,1 & 89 & 53,6 & 0,96 & $0,67-1,39$ & 0,830 \\
\hline Irregularmente ativos "A" & 60 & 8,7 & 20 & 12,0 & 1,24 & $0,73-2,19$ & 0,421 \\
\hline Irregularmente ativos "B" & 61 & 8,9 & 8 & 4,8 & 0,58 & $0,27-1,23$ & 0,154 \\
\hline Sedentários & 28 & 4,1 & 7 & 4,2 & 0,99 & $0,45-2,21$ & 0,991 \\
\hline \multirow{2}{*}{\multicolumn{8}{|c|}{$\begin{array}{l}\text { Frequência de participação nas aulas de } \\
\text { Educação Física escolar }\end{array}$}} \\
\hline & & & & & & & \\
\hline Sempre & 257 & 37,4 & 54 & 32,5 & ref. & ref. & ref. \\
\hline Às vezes & 335 & 48,7 & 84 & 50,6 & 0,87 & $0,61-1,22$ & 0,410 \\
\hline Nunca & 96 & 14,0 & 28 & 16,9 & 1,13 & $0,73-1,73$ & 0,556 \\
\hline \multicolumn{8}{|l|}{ Obesidade $^{*}$} \\
\hline Magros & 79 & 11,5 & 9 & 5,4 & 0,61 & $0,31-1,21$ & 0,156 \\
\hline Eutrófico & 487 & 70,8 & 98 & 59,0 & ref. & ref. & ref. \\
\hline Sobrepeso & 25 & 3,6 & 22 & 13,3 & 1,65 & $1,13-2,41$ & 0,010 \\
\hline Obeso & 97 & 14,1 & 37 & 22,3 & 2,79 & $1,76-4,44$ & 0,000 \\
\hline \multicolumn{8}{|l|}{ IDH do bairro da escola } \\
\hline Muito elevado & 261 & 37,9 & 65 & 39,2 & ref. & ref. & ref. \\
\hline Elevado & 300 & 43,6 & 75 & 45,2 & 1,00 & $0,72-1,40$ & 0,985 \\
\hline Médio & 127 & 18,5 & 26 & 15,7 & 0,85 & $0,54-1,34$ & 0,491 \\
\hline \multicolumn{8}{|l|}{ Coordenadoria Metropolitana } \\
\hline Metropolitana III & 290 & 42,2 & 62 & 37,3 & ref. & ref. & ref. \\
\hline Metropolitana IV & 197 & 28,6 & 51 & 30,7 & 1,17 & $0,81-1,69$ & 0,413 \\
\hline Metropolitana X & 201 & 29,2 & 53 & 31,9 & 1,19 & $0,82-1,71$ & 0,365 \\
\hline \multicolumn{8}{|l|}{ Classificação sócio-econômica } \\
\hline Classe "A" & 21 & 3,1 & 1 & 0,6 & ref. & ref. & ref. \\
\hline Classe “B” & 282 & 41,0 & 78 & 47,0 & 4,77 & $0,66-34,26$ & 0,121 \\
\hline Classe "C" & 349 & 50,7 & 82 & 49,4 & 4,18 & $0,58-30,07$ & 0,155 \\
\hline Classes “D” e “E” & 36 & 5,2 & 5 & 3,0 & 2,68 & $0,31-22,96$ & 0,368 \\
\hline
\end{tabular}

RP - razão de prevalência; IC - intervalo de confiança; ref - referência; IDH - índice de desenvolvimento humano; IPAQ Questionário Internacional de Atividade Física; ${ }^{*}$ Associação significativa $(\mathrm{p}<0,05)$.

Outras lacunas dentro desse contexto investigativo ratificam a importância dos resultados aqui descritos. Primeiro a escassez de investiga- 


\begin{tabular}{|c|c|c|c|c|c|c|c|c|}
\hline \multirow[t]{10}{*}{ 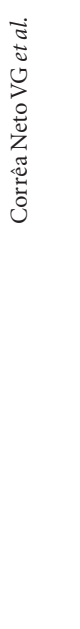 } & $\begin{array}{l}\text { Tabela 4. Fatores de } \\
\text { médio investigados }\end{array}$ & $\begin{array}{l}\text { ados à pı } \\
\text { tivariado }\end{array}$ & valência d & pertens & o arter & re os es & antes do en & no \\
\hline & \multirow[t]{2}{*}{ Variáveis } & \multicolumn{2}{|c|}{ Não hipertensos } & \multicolumn{2}{|c|}{ Hipertensos } & \multirow[b]{2}{*}{$\mathbf{R P}$} & \multirow[b]{2}{*}{ IC } & \multirow[b]{2}{*}{$\mathbf{p}$} \\
\hline & & $\mathrm{n}$ & $\%$ & $\mathrm{n}$ & $\%$ & & & \\
\hline & \multicolumn{8}{|l|}{ Sexo $^{*}$} \\
\hline & Meninas & 412 & 59,9 & 68 & 41 & ref. & ref. & ref. \\
\hline & Meninos & 276 & 40,1 & 98 & 59 & 1,87 & $1,37-2,56$ & 0,000 \\
\hline & \multicolumn{8}{|l|}{ Obesidade $^{*}$} \\
\hline & Eutrófico & 487 & 70,8 & 98 & 59,0 & ref. & ref. & ref. \\
\hline & Sobrepeso & 25 & 3,6 & 22 & 13,3 & 1,66 & $1,13-2,42$ & 0,009 \\
\hline & Obeso & 97 & 14,1 & 37 & 22,3 & 2,91 & $1,83-4,63$ & 0,000 \\
\hline
\end{tabular}

RP - razão de prevalência; IC - intervalo de confiança; ref - referência; ${ }^{\star}$ Associação significativa $(\mathrm{p}<0,05)$.

formações para a população aqui representada. A falta de maiores explicações a respeito do calculo amostral, configura um sucessivo erro em estudos epidemiológicos e isso limita a extrapolação de seus achados.

Sexo masculino mostrou uma significativa associação com HAS. Em verdade outros estudos corroboram esse achado em jovens ${ }^{19,23,24}$. Dasgupta et al. ${ }^{25}$ acompanharam uma coorte de adolescentes durante cinco anos e também relatam um maior risco no aumento da pressão arterial sistólica vinculada ao sexo masculino. $\mathrm{O}$ referido estudo discute a possibilidade de que esteroides sexuais poderiam ter efeito nos valores pressóricos ao longo da adolescência. $\mathrm{O}$ aumento dos níveis de testosterona no sexo mascu$\operatorname{lino}^{26}$, bem como seu possível efeito pró-hipertensivo $^{27}$ são o embasamento fisiológico para tais especulações. Porém, a estrutura metodológica do presente estudo, não incluiu dosagem hormonal, impossibilitando a ratificação de tais hipóteses, embora, pertinentemente amparem os resultados aqui relatados.

No que diz respeito às associações entre obesidade e HAS, os resultados apresentados corroboram com o conceito hegemônico de associação positiva entre sobrepeso e obesidade com níveis pressóricos ${ }^{5}$. Embora tal paradigma tenha ampla aderência da comunidade acadêmica, pontuar certas particularidades em relação a tal discurso pode ser interessante. Outros estudos que também empregaram o IMC como instrumento de categorização da obesidade, verificaram associações de cunho negativo com as cifras pressóricas $^{28,29}$. A literatura ilustra também associação de âmbito negativo entre razão cintura quadril e níveis pressóricos ${ }^{30}$, e ainda uma série de outras investigações identificou ausência de associação significativa entre HAS e outras medidas referen- tes à obesidade como circunferência de cintura ${ }^{19}$, dobras cutâneas ${ }^{31}$ e percentual de gordura ${ }^{32}$. Sendo assim, o cenário conflitante dos resultados científicos, embasa uma situação de incerteza do conhecimento, e põe em pauta a premissa dos valores hegemônicos sobre obesidade, que pode se amparar muito mais sobre égides morais do que sobre evidências acadêmicas ${ }^{33}$.

Embora a visão de causa e efeito advogue a favor do risco da obesidade em relação às condições pressóricas, interessantemente, Uretsk et al..$^{34}$ observaram um efeito protetor de maiores valores de IMC sobre condições de morbidade e mortalidade em sujeitos hipertensos, configurando assim um retrato paradoxal de tais relações.

Deve-se acrescentar que o uso do IMC como método avaliador da obesidade, poderia retratar uma fragilidade do estudo. Porém, não se tentou aqui, discursar sobre prevalência de obesidade, e sim, sobre suas associações com PA. Para tal aplicabilidade em grupos de indivíduos jovens o IMC vem se mostrando mais relevante do que outras medidas de composição corporal ${ }^{1,35}$.

A relevância do IMC nos valores pressóricos de jovens faz beirar a desconstrução de um conceito hegemônico que prega a favor dos efeitos deletérios do excesso de gordura corporal em variáveis de ordem cardiovascular. As informações fornecidas pelo IMC não permitem conhecimento de ordem da composição corporal, porém, mesmo assim, tal índice, se mostrou uma ferramenta mais robusta na predição de níveis pressóricos, do que outros instrumentos como dobras cutâneas e circunferência de cintura ${ }^{35}$.

No grupo amostral aqui relatado, a atividade física não demonstrou efeito protetor sobre os padrões pressóricos. A falta de associação entre atividade física e HAS já foi ilustrada em outros estudos com jovens ${ }^{7,19,36}$, e, muito embora a 
crença de que maiores níveis de atividade física possuiriam um efeito cardioprotetor, no que diz respeito a cifras pressóricas de jovens os resultados não são consensuais ${ }^{37}$. $\mathrm{O}$ tempo semanal aceito, via de regra, como ponto de corte para caracterizar adolescentes quanto a seus hábitos de atividade física são 300 minutos $^{38}$. Os resultados aqui descritos não corroboram tal quantificação no que diz respeito ao possível efeito protetor da prática de exercícios sobre níveis de PA. Ao contrário, embora, a associação não tenha sido significativa para nenhuma das categorizações de tempo, se tendências fossem levadas em consideração, pode-se dizer que os sujeitos que declararam não realizar atividades físicas desportivas, mostraram uma forte tendência protetora de tal hábito diante da HAS ( $\mathrm{p}=0,051)$.

Alomari et al..$^{39}$ também utilizaram o IPAQ na tentativa de relacionar níveis de atividade física de jovens com valores pressóricos. Os autores encontraram correlações de cunho negativo e significativo entre atividade física e PA. Porém, alguns aspectos pertinentes à referida investigação merecem ser pontuados. A amostra pesquisada pelos autores era composta inteiramente por sujeitos normotensos, sendo assim, pode-se dizer que existiu uma correlação entre atividade física e $\mathrm{PA}$, porém, não entre atividade física e HAS. A extrapolação dos resultados para um grupo hipertenso seria um tanto quanto arbitrária, pois assumir-se-ia que hipertensos e normotensos, reagiriam da mesma forma à pratica de atividade física, e desprezar-se-ia fatores genéticos que poderiam ter relevante contribuição nas características do grupo amostral, tanto referente aos valores de PA, quanto das adaptações cardiovasculares ao exercício.

Wisloff et al. ${ }^{40}$, a partir de 11 gerações de ratos, selecionaram animais com maior capacidade aeróbia geneticamente e animais com capacidade aeróbia desprivilegiada geneticamente. Os autores puderam perceber que antes do treinamento, os ratos menos dotados geneticamente possuíam fatores de risco cardiovasculares mais proeminentes do que seus pares geneticamente privilegiados. Após o treinamento, pôde-se notar que a adaptação dos ratos geneticamente privilegiados, no que diz respeito às variáveis cardiovasculares, foi de maior magnitude do que dos ratos inaptos geneticamente. Tal estudo, claramente denota que a capacidade de adaptação crônica ao exercício físico parece sofrer influência direta de melhores condições genéticas, e põe em xeque qualquer tentativa de extrapolação de dados de forma similar entre grupos que apresentam características de base antagônicas referentes aos padrões cardiovasculares. Alomari et al..$^{39}$ não trabalharam com sujeitos hipertensos. Essa limitação metodológica inviabiliza extrapolações da correlação negativa entre atividade física e PA encontrada pelos autores para sujeitos hipertensos.

Ainda, o teste de correlação, medida utilizada no estudo em questão para ilustrar a relação entre atividade física e PA, é uma técnica estatística incapaz de ilustrar as associações de risco entre expostos, não expostos e determinado desfecho. Tais relações nem sempre são lineares e tal método pode não ser capaz de identificar relações não lineares ${ }^{41}$.

Uma reflexão mais profunda sobre as relaç̃es entre atividade física e fatores cardioprotetores deveria ser levada em consideração quando da análise das respostas de âmbito biológico. Apesar da ideia protecionista da atividade física encontrar ampla adesão, alguns achados colocam em conflito a veracidade de tais conceitos. Albert et al. ${ }^{42}$, por exemplo, ilustram um quadro paradoxal. Tal investigação mostrou que o exercício extenuante apresentava um maior impacto enquanto medida cardioprotetora, ao mesmo tempo em que exibia um significativo risco relativo para morte súbita.

Vale ressaltar que recomendações a respeito de cuidados diagnósticos de HAS se mostraram fracamente representados em nossa amostra. Recomenda-se a mensuração da PA em crianças a partir dos três anos em consultas médicas ${ }^{5}$. Entretanto 40,7\% do grupo aqui analisado nunca haviam medido a PA antes, impossibilitando o diagnóstico e por consequência o tratamento precoce de tal condição.

É interessante ressaltar algumas possíveis fragilidades do estudo. O caráter metodológico transversal impossibilita a inferência de relações causais aos achados aqui descritos, muito embora tal desenho tenha relevância no que diz respeito às informações no âmbito de caracterização populacional e levantamento de possíveis fatores de risco. O uso de instrumentos self report para a coleta de determinadas variáveis também poderia ser apontado como um ponto fraco da investigação $0^{43}$, porém, dentre tais instrumentos, se buscou aplicar os que já previamente teriam apresentados boa fidedignidade dentro desse contexto etário.

Ainda, algumas características metodológicas zelam pela relevância das informações aqui relatadas. A quantificação da amostra feita de maneira probabilística confere a possibilidade de extrapolação dos resultados para a população aqui representada. 
O tempo de coleta de dados também reforça a credibilidade das informações do estudo, já que todo processo durou apenas três meses. Tal estratégia torna viável a possibilidade de minimizar os efeitos da sazonalidade de certas informações, como pratica de atividade física, que pode ter ampla variação durante o ano.

\section{Conclusão}

A prevalência de HAS em adolescentes da cidade do Rio de Janeiro foi de 19,4\%. Tal número re- presenta um alto valor quantitativo da referida variável em tal contexto etário. Ainda, sobrepeso e obesidade apresentaram associação positiva e significativa com HAS, porém, tal resultado não deveria ser passivamente analisado, e sim interpretado sob uma ótica critica de tais relações, já que uma série de evidências científicas parecem fragilizar a relação causal entre esse tipo de associação. A atividade física, não foi capaz de apresentar associação significativa com HAS. Esse resultado vem robustecer o conflito de evidências sobre os possíveis efeitos protetores do exercício sobre níveis pressóricos de jovens.

\section{Colaboradores}

VG Corrêa Neto participou da concepção e do delineamento do estudo, da coleta, análise e interpretação dos dados, da redação, revisão crítica e aprovação da versão a ser publicada. S Sperandei participou da análise e interpretação dos dados, análise estatística e aprovação da versão final a ser publicada. LAI Silva e GA Maranhão Neto participaram da concepção e delineamento do estudo, análise e interpretação dos dados e aprovação da versão a ser publicada. A Palma participou da concepção e delineamento do estudo, análise e interpretação dos dados, redação e revisão crítica e aprovação da versão a ser publicada. 


\section{Referências}

1. Wang H, Necheles J, Carnethon M, Wang B, Li Z, Wang L, Liu X, Yang J, Tang G, Xing H, Xu X, Wang $\mathrm{X}$. Adiposity measures and blood pressure in Chinese children and adolescents. Arch Dis Child 2008; 93(9):738-744

2. Hajjar I, Kotchen TA. Trends in prevalence, awareness, treatment and control of Hypertension in the United States, 1988-2000. JAMA 2003; 290(2):199206.

3. Lewington S, Clarke L, Qizilbash N, Peto R, Collins R. Age-specific relevance of usual blood pressure to vascular mortality: a meta-analysis of individual data for one million adults in 61 prospective studies. Lancet 2002; 360(9349):1903-1913.

4. Din-Dzietham R, Liu Y, Bielo M, Shamsa F. High blood pressure trends in children and adolescents in national surveys, 1963 to 2002. Circulation 2007; 116(13):1488-1496.

5. American Academy of Pediatrics. The fourth report on the diagnosis, evaluation, and treatment of high blood pressure in children and adolescents. Pediatrics 2004; 114(2 Supl. $4^{\text {th }}$ Report):555-576.

6. Daniels SR, Pratt CA, Hayman LL. Reduction of risk for cardiovascular disease in children and adolescents. Circulation 2011; 124(15):1673-1686.

7. Gaya AR, Silva P, Martins C, Gaya A, Ribeiro JC, Mota J. Association of leisure time physical activity and sports competition activities with high blood pressure levels: Study carried out in a sample of Portuguese children and adolescents. Child Care Health Dev 2011; 37(3):329-334.

8. Torrance B, McGuire KA, Lewanczuk R, McGavock J. Overweight, physical activity and high blood pressure in children: a review of the literature. Vas Health Risk Manag 2007; 3(1):139-149.

9. Christofaro DGD, Fernandes RA, Gerage AM, Alves MJ, Polito MD, Oliveira AR. Validação do monitor de pressão arterial Omron HEM 742 em adolescentes. Arq Bras Cardiol 2009; 92(1):10-15.

10. Guedes DP, Lopes CC, Guedes ERP. Reprodutibilidade e validade do questionário internacional de atividade física em adolescentes. Rev Bras Med Esporte 2005; 11(2):151-158.

11. Cole TJ, Bellizzi MC, Flegal KM, Dietz WH. Establishing a standard definition for child overweight and obesity worldwide: international survey. $B M J$ 2000; 320(7244):1240-1243.

12. Cole TJ, Flegal KM, Nicholls D, Jackson AA. Body mass index cut offs to define thinness in children and adolescents. BMJ 2007; 335(7612):194-201.

13. Pickering TG, Hall JE, Appel LJ, Falkner BE, Graves J, Hill MN, Jones DW, Kurtz T, Sheps SG, Roccella EJ; Subcommittee of Professional and Public Education of the American Heart Association Council on High Blood Pressure Research. Recommendations for blood pressure measurement in humans and experimental animals: Part 1: blood pressure measurement in humans: a statement for professionals from the Subcommittee of professional and public education of the American heart association council on high blood pressure research. Hypertension 2005; 45(1):142-161.
14. Sociedade Brasileira de Cardiologia. Diretrizes Brasileiras de Hipertensão Arterial. Arq Bras Cardio 2007; 89(3):e24-e79.

15. Cifkova R, Erdini S, Fagard R, Farsang C, Heargerty AM, Kiowski W, Kjeldsen S, Lüscher T, Mallion JM, Mancia G, Poulter N, Rahn KH, Rodicio JL, Ruilope LM, van Zwieten P, Waeber B, Williams B, Zanchetti A; ESH/ESC Hypertension Guidelines Committee. Practice guidelines for primary care physicians: 2003 ESH/ESC Hypertension guidelines. J Hypertens 2003; 21(10):1779-1786.

16. Brasil. Ministério da Saúde (MS). Conselho Nacional de Saúde. Resolução no 196 de 10 de outubro de 1996. Diretrizes e Normas Regulamentadoras de Pesquisas Envolvendo Seres Humanos. Diário Oficial da União 1996; 16 out.

7. Flynn JT, Falkner BE. Obesity Hypertension in adolescents: epidemiology, evaluation, and management. J Clin Hypertens 2011; 13(5):323-331.

18. Pileggi C, Carbone V, Nobile CGA, Pavia M. Blood pressure and related cardiovascular disease risk factors in 6-18 year-old students in Italy. $J$ Paediatr Child Health 2005; 41(7):347-352.

9. Monego ET, Jardim PCBV. Determinants of risk of cardiovascular diseases in schoolchildren. Arq Bras Cardiol 2006; 87(1):37-45.

20. Aounallah-Skhiri H, El Ati J, Traissac P, Ben Romdhane $\mathrm{H}$, Eymard-Duvernay S, Delpeuch F, Achour $\mathrm{N}$, Maire B. Blood pressure and associated factors in a north African adolescent population. A national cross-sectional study in Tunisia. BMC Public Health 2012; 12:98.

21. Chiolero A, Bovet P, Paredis G, Paccaud F. Has blood pressure increased in children in response to the obesity epidemic? Pediatrics 2007; 119(3):544-553.

22. Ribeiro RQC, Lotufo PA, Lamounier JA, Oliveira RG, Soares JF, Botter DA. Additional cardiovascular risk factors associated with excess weigth in children and adolescents. The Belo Horizonte study. Arq Bras Cardiol 2006; 86(6):406-415.

23. Kelishadi R, Ardalan G, Gheiratmand R, Majdzadeh R, Delavari A, Heshmat R, Gouya MM, Razaghi EM, Motaghian M, Mokhtari MR, Barekati H, Arabi MS; CASPIAN Study Group. Blood pressure and its influencing factors in a national representative sample of Iranian children and adolescents: the CASPIAN Study. Eur J Cardiovasc Prev Rehabil 2006; 13(6):956-963

24. Leung LCK, Sung RYT, So HK, Wong SN, Lee KW, Lee KP, Yam MC, Li SP, Yuen SF, Chim S, Chan KK, Luk D. Prevalence and risk factors for Hypertension in Hong Kong Chinese adolescents: waist circumference predicts Hypertension, exercise decrease risk. Arch Dis Child 2011; 96(9):804-809.

5. Dasgupta K, O'Loughlin J, Chen S, Karp I, Paradis G, Tremblay J, Hamet P, Pilote L. Emergence of sexes differences in prevalence of high systolic blood pressure. Circulation 2006; 114(24):2663-2670.

26. Ré AHN. Crescimento, maturação e desenvolvimento na infância e adolescência: implicações para o esporte. Motricidade 2011; 7(3):55-67. 
27. Dubey RK, Oparil S, Imthurn P, Jackson EK. Sex hormones and Hypertension. Cardiovascular Research 2002; 53(3):688-708.

28. Sugyiama T, Xie D, Graham-Maar RC, Inoue K, Kobayashi Y, Stettler N. Dietary and lifestyle factors associated with blood pressure among U.S. adolescents. J Adolesc Health 2007; 40(2):166-172.

29. Forrest KYZ, Leeds MJ. Prevalence and associated factors of overweight among Mexican-American adolescents. J Am Diet Assoc 2007; 107(10):1797-1800.

30. Burgos MS, Reuter CP, Burgos LT, Pohl HH, Pauli LTS, Horta JA, Reckziegel MB, Franke SI, Prá D, Camargo M. Comparison analysis of blood pressure, obesity and cardio-respiratory fitness in schoolchildren. Arq Bras Cardiol 2010; 94(6):739-744.

31. Monyeki KD, Kemper HCG, Makgae PJ. The association of fat patterning with blood pressure in rural south African children: the Ellisras longitudinal growth and health study. Int J Epidemiol 2006; 35(1):114-120.

32. Ribeiro JC, Guerra S, Oliveira J, Teixeira-Pinto A, Twisk JWR, Duarte JA, Mota J. Physical activity and biological risk factors clustering in pediatric population. Prev Med 2004; 39(3):596-601.

33. Campos P, Saguy A, Ernsberger P, Oliver E, Gaesser G. The epidemiology of overweight and obesity: public health crisis or moral panic? Int J Epidemiol 2006; 35(1):55-60

34. Uretsky S, Messerli FH, Bangalore S, Champion A, Cooper-Dehoff RM, Zhou Q, Pepine CJ. Obesity paradox in patients with Hypertension and coronary artery disease. Am J Med 2007; 120(10):863-870.

35. Ma J, Wang Z, Dong B, Song Y, Hu P, Zhang B. Body fat and blood pressure: comparison of blood pressure measurements in Chinese children with different body fat levels. Br J Nutr 2012; 108(9):1672-1677.

36. Silva DAS, Lima RLA, Dellagrana RLA, Bacil EDA, Rech CR. Pressão arterial elevada em adolescentes: prevalência e fatores associados. Cien Saude Colet 2013; 18(11):3391-3400.

37. Pescatello LS, Franklin BA, Fagard R, Farquhar WB, Kelley GA, Ray CH. Exercise and Hypertension. Med Sci Sport Exer 2004; 36(3):533-553.
38. Hallal PC, Wells JCK, Reichert FF, Anselmi L, Victora CG. Early determinants of physical activity in adolescence: prospective birth cohort study. BMJ 2006; 332(7548):1002-1007.

39. Alomari MA, Keewan EF, Qhatan R, Amer A, Khabour OF, Maayah MF, Hurtig-Wennlöf A. Blood pressure and circulatory relationships with physical activity level in young normotensive individuals: IPAQ validity and reliability considerations. Clin Exp Hypertens 2011; 33(5):345-353.

40. Wisloff U, Najjar SM, Ellingsen O, Haram PM, Swoap S, Al-Share Q, Fernström M, Rezaei K, Lee SJ, Koch LG, Britton SL. Cardiovascular risk factors emerge after artificial selection for low aerobic capacity. Science 2005; 307(5708):418-420.

41. Freedman DS, Dietz WH, Srinivasan SR, Berenson GS. The relation of overweight to cardiovascular risk factors among children and adolescents: The Bogalusa Heart Study. Pediatrics 1999; 103(6 Pt 1):1175-1182.

42. Albert CM, Mittleman MA, Chae CU, Lee M, Hennekens $\mathrm{CH}$, Manson JE. Triggering of sudden death from cardiac causes by vigorous exertion. $N$ Engl $J$ Med 2000; 343(19):1355-1361.

43. Romero A, Slater B, Florindo AA, Latorre MRDO, Cezar C, Silva MV. Determinantes do índice de massa corporal em adolescentes de escolas públicas de Piracicaba, São Paulo. Cien Saude Colet 2010; 15(1):141-149.

Artigo apresentado em 27/04/2013

Aprovado em 04/06/2013

Versão final apresentada em 17/06/2013 